\title{
Diffusion of nanoparticles in dense fluids
}

\author{
F. Ould-Kaddour \\ Laboratoire de Physique Théorique, Faculté des Sciences, \\ Université de Tlemcen BP 119, Tlemcen 13000 Algerie \\ D. Levesque \\ Laboratoire de Physique Théorique, Université Paris-Sud, \\ Bâtiment 210, 91405 Orsay, France*
}

\begin{abstract}
The diffusion process of a single spherical nanoparticle immersed in a fluid solvent is studied by molecular dynamics simulations. When the nanoparticle mass stays constant, it is shown that, at short times, the decay of the nanoparticle velocity autocorrelation function is strongly modified when the particle diameter increases. It is also shown that, at large times, the characteristic algebraic decay induced by the hydrodynamic correlations between the solvated particle and the solvent presents a scaling behavior depending on the particle diameter.
\end{abstract}

PACS numbers: 05.10.-a, 61.25.-f 


\section{INTRODUCTION}

The determination of transport properties of particles with a nanometric size immersed in a solvent is of large interest for the present researches in the domain of nanotechnologies ${ }^{1}$. When the nanoparticles have a diameter larger than $100 \mathrm{~nm}$ and, hence, a mass larger by several orders of magnitude than that of the solvent particles, they can be considered in practical applications as brownian particles ${ }^{2}$. However when the size and mass of nanoparticles differ from those of the solvent particles only by a factor $\sim 10$, the transport properties of such particles could differ from those expected for brownian particles. Examples of this type of nanoparticles are the fullerenes, the carbon nanotubes of short length, ... etc ... .

Numerous theoretical works have been published in the literature on this subject by using molecular dynamics simulations. Many of these studies ${ }^{3-9}$ have been devoted to determine the size and mass of solvated particles at which, in the low concentration limit, the diffusion coefficient $D$ is well estimated by the Stokes-Einstein relation $D=k_{B} T /\left(\alpha_{h} \pi \eta R\right)$ where $k_{B}, T, \eta$ and $R$ are the Boltzmann constant, the solvent temperature, the solvent viscosity and the radius of the solvated particle, respectively. $\alpha_{h}$ is a numerical coefficient determined by the choice of the hydrodynamic limit condition at the interface between the fluid solvent and the solvated particle. An unambiguous conclusion on this point is not easy to achieve, because the Stokes-Einstein relation derives from the Einstein relation $D=k_{B} T / \xi$, where $\xi$, the friction coefficient, is well defined only in the brownian limit as the integration of the time autocorrelation function of the force acting on the brownian particle ${ }^{6,10,11}$. Then, the use of the Stokes-Einstein relation a priori should suppose that two conditions are fulfilled : brownian limit and validity of a hydrodynamic estimate of $\xi^{2,12}$.

Briefly summarized, the conclusions of these works are that the Stokes-Einstein relation can give a qualitatively acceptable estimate of the diffusion coefficient even if the two conditions are not satisfied. But when a quantitative estimate is required, $\alpha_{h}$ must be considered as a free parameter and, for instance, adjusted between the values of 4 (slip boundary condition) and 6 (stick boundary condition). Such an adjustment of $\alpha_{h}$ values is obviously needed to take into account the approximate character of the Stokes-Einstein and Einstein relations when the size and mass of the tracer particles are insufficiently large with respect to those of the solvent particles.

In order to investigate the specific case where the mass and size ratios between solvated 
and solvent particles are smaller than 100, we will consider a model in which the mass ratio $m_{t} / m_{s}$ is equal to $60\left(m_{t}\right.$ and $m_{s}$ : mass of the solvated and solvent particles, respectively) and the size ratio varies between 1 and 17 . Such a model where the mass $m_{t}$ of solvated particles is kept constant while their size varies, is chosen in order to determine the qualitative changes on the nanoparticle diffusion only induced by the increase of the nanoparticle diameter. We study the case of the low concentration limit of the solvated nanoparticles, i.e. concentrations smaller than $0.510^{-4}$. Our aim is to obtain by molecular dynamics (MD) simulations at constant energy (microcanonical ensemble) a detailed description of the diffusion process, in particular, as mentioned above, how this process varies with the size of the solvated particle, through the computation of the velocity autocorrelation function (VAF) $c(t)$

$c(t)=\frac{1}{t_{m}-t} \int_{0}^{t_{m}-t} d \tau \mathbf{v}(\tau) \cdot \mathbf{v}(\tau+t)=c(0) z(t)=\frac{3 k_{\mathrm{B}} T}{m_{t}} z(t)$,

where $\mathbf{v}(t)$ is the nanoparticle velocity, $z(t)$ the normalized VAF and $t_{m}$ the total integration time of the motion equations in a simulation run.

In Sec. II, the studied system and simulation procedure are described and the simulations data are presented. Sec. III is devoted to the analysis of the results and the discussion of proposed corrections to take into account the finite size effects inherent to simulation data. In the last section, the conclusions of the analysis will be summarized with a special mention of the scaling behavior of the VAF long-time tail with the solvated particle diameter.

\section{SYSTEMS AND SIMULATIONS}

Similarly to models used in our previous works ${ }^{9,10}$ or works published in the literature, the studied system is composed of $N$ solvent particles and one solvated particle. The solvent particles and, also the solvated particle with the solvent particles, interact by a truncated soft repulsive Lennard-Jones (LJ) pair potential :

$$
\begin{aligned}
v\left(r_{i j}\right) & =v_{L J}\left(r_{i j}\right)+\epsilon=4 \epsilon\left[\left(\frac{\sigma_{\alpha \beta}}{r_{i j}}\right)^{12}-\left(\frac{\sigma_{\alpha \beta}}{r_{i j}}\right)^{6}\right]+\epsilon \quad \text { for } \quad r_{i j} \leq 2^{\frac{1}{6}} \sigma_{\alpha \beta} \\
& =0 \text { for } \quad r_{i j}>2^{\frac{1}{6}} \sigma_{\alpha \beta}, \quad \alpha, \beta=0 \text { or } 1,
\end{aligned}
$$

where $r_{i j}$ is the distance between solvent or tracer particles $i$ and $j . \sigma_{11}$ is the LJ size parameter of the solvent particles, $\sigma_{00}$ that of the solvated particle and, for the cross LJ pair 
potential, $\sigma_{01}$ is given by $\sigma_{01}=0.5\left(\sigma_{11}+\sigma_{00}\right)$. The choice of these spherical, repulsive and short ranged LJ potentials is suitable for the main purpose of our study (cf. Introduction). When $\sigma_{00}$, defining the size of the solvated particle varies the solvent thermodynamic state and $m_{t}$ staying identical, the modifications of the solvated particle diffusion clearly depend unambiguously on this only parameter. $\sigma_{11}$ and $\epsilon$ being chosen as the units of length and energy, the time unit $\tau_{0}$ is $\sqrt{m_{s} \sigma_{11}^{2} / \epsilon}$.

The computation of the asymptotic behavior of the tracer VAF requires to perform the simulations with systems large enough to allow the existence of a time domain where this behavior is not affected by the coupling between the hydrodynamic diffusive and sound modes, resulting from the periodic boundary conditions of the simulation cell ${ }^{13}$. In addition, the system size having to be sufficient to accomodate a large solvated particle, the simulations are realized for $N=55296$ when $\sigma_{00}$ is equal to 1,4 and 7 and $N=87808$ when $\sigma_{00}$ is equal to 11 and 17. The solvent density in reduced units is $\rho_{s} \sigma_{11}^{3}=0.2$, so that the sides $L_{c} / \sigma_{11}$ of the simulation cubic cells are equal to $65.15(N=55296)$ or $76.13(N=87808)$. With these $L_{c}$ values, at a temperature $T^{*}=k_{B} T / \epsilon=2.01$ where the sound velocity $c_{s}$ is equal to $2.68^{14}$, it is expected that the VAF asymptotic behavior can be computed until $t_{c}=L_{c} / c_{s}$ equal to $24.3 \tau_{0}$ and $28.6 \tau_{0}$ for the systems with $N=55296$ and $N=87808$, respectively. It is probably more realistic to take into account the size of the solvated particle and to compute $t_{c}$ from $t_{c}=\left(L_{c}-\sigma_{00}\right) / c_{s}$, giving $t_{c} \simeq 21.0 \tau_{0}$ and $\simeq 22.0 \tau_{0}$ for the small $\left(\sigma_{00}=4\right.$ and 7$)$ and large $\left(\sigma_{00}=11\right.$ and 17$)$ sizes of the tracer particle.

The computation of the VAF of one isolated particle in a solvent needs very extensive simulations, peculiarly when an accuracy of $\sim 10 \%$ is required on the values of the VAF asymptotic behavior which is expected to vary with $t$ as $\alpha_{s} t^{-3 / 2} 15,16$. In order to achieve such an accuracy, 67 runs of $10^{5}$ integration time steps $\left(\Delta t=0.005 \tau_{0}\right)$ have been realized for computing the nanoparticle VAF for $\sigma_{00}=1,1020$ runs of $10^{5} \Delta t$ for $\sigma_{00}=4,1790$ runs of $50 \times 10^{3} \Delta t$ for $\sigma_{00}=7,2900$ runs of $50 \times 10^{3} \Delta t$ for $\sigma_{00}=11$ and 2050 runs of $50 \times 10^{3} \Delta t$ for $\sigma_{00}=17$. $c(t)$ was computed up to $30.0 \tau_{0}$ in order to determine the time domain where its long-time behavior becomes proportional to $t^{-3 / 2}$. The statistical error on the VAF was obtained by calculating a standard error from the estimates of $c(t)$ computed after each period of 3000 time steps during the MD runs.

The results of these simulations are presented in Fig. 1 in the time domain where the function $z(t)=c(t) / c(0)$ is expected to be without bias induced by the coupling with the 
hydrodynamic sound modes. For $t / \tau_{0}<2.0$, the estimated statistical error is about $1-2 \%$ for all particles diameters but it increases from $1-2 \%$ up to $10-15 \%$ at $t / \tau_{0}>5.0$ when $\sigma_{00}$ varies from 1 to 17 .

\section{ANALYSIS AND DISCUSSIONS}

At short times, defined as $t / \tau_{0}<5.0, z(t)$ varies from an exponential or quasi-exponential decrease for $\sigma_{00}=1$, to a more complex variation involving two disctinct rates of decrease, clearly visible at $\sigma_{00}=11$, and, finally, to an oscillatory behavior at $\sigma_{00}=17$ (cf. Fig. 1). For $\sigma_{00}=1$, within the statistical error, a simple exponential $c \exp \left(-\alpha_{z} t\right)$ gives an excellent curve fitting of $z(t)$ on the time domain from 0.2 to 5.0 with $\alpha_{z}=0.030$, (cf. Fig. 2). This exponential decay continues up to $t=22.0 \tau_{0}$, but differs from that expected if the diffusion of the particle was of brownian type satisfying to the Stokes-Einstein relation. In this case, $\alpha_{z}$ would be given by $\alpha_{h} \pi m_{s} \rho_{s} \nu R / m_{t}{ }^{17}$, with $\nu=\eta / m_{s} \rho_{s}$ and $m_{t}$ the mass of the solvated particle, and would be equal to $0.035\left(\alpha_{h}=4\right)$ or $0.053\left(\alpha_{h}=6\right), \nu$ being equal to 1.71 in reduced units ${ }^{14}$. These quantitative differences are typical of those found in the test of the validity of the Stokes-Einstein relation (cf. above), if it is supposed that $c(t)$ keeps for $t$ larger than $t=22.0 \tau_{0}$ the exponential behavior determined from its short-time values for $t / \tau_{0}<5$.

When the solvated particle has a diameter $\sigma_{00}=4,7$ or 11, a fit of $z(t)$ by a single exponential in the time domain $0<t / \tau_{0}<5.0$ is not possible within the estimated statistical errors. Fits realized with a linear combination of two exponentials allow to show that the fast decrease for $t / \tau_{0}<2.0$, is followed by a more slow decrease ${ }^{18}$. But in contrast with the result obtained for $\sigma_{00}=1$ with one exponential, the values of the parameters are very sensitive to the upper bound of the time interval chosen to realize the fits so that the identification of two well characterized regimes of exponential decrease in the range $0<t / \tau_{0}<10$ is precluded. A fortiori, a similar result is obtained at $\sigma_{00}=17$, where $z(t)$ presents an oscillatory behavior, indicating an effect of backscattering on the solvated particle due to the solvent, followed by a slow monotonic decrease. From the previous discussion, it appears that, at short times, the diffusion process differs strongly with the $\sigma_{00}$ values. In this time range, $z(t)$ goes from an exponential decrease similar to that of brownian particle to a damped oscillatory decrease similar to that obtained for particles in a dense fluid. 
For the time domain $\left(12<t / \tau_{0}<18\right)$ where the sound mode should be without influence on the diffusion process and excluding the case $\sigma_{00}=1$ where, for these $t$ values, the $z(t)$ decrease stays exponential, it is seen in Fig. 3 that $z(t)$ is fitted within the statistical error by the expected analytic form $\alpha_{s} t^{-3 / 2}$. The fits are very accurate for $\sigma_{00}=4,7$ and 11 , for $\sigma_{00}=17$ the fit stays acceptable, but the MD data are affected by an important statistical noise. The fits, realized with a two-parameter form $\alpha_{s} t^{-a_{0}}$, give values of $a_{0}$ close of $3 / 2$ : $1.48,1.52$ and 1.58 for $\sigma_{00}$ equal to 4,7 and 11 , respectively, while the $\alpha_{s}$ values differ by $10-15 \%$ from the values obtained when $a_{0}$ is equal to $3 / 2$. For $\sigma_{00}=17$, the large statistical errors make the variations on $\alpha_{s}$ and $a_{0}$ much larger, i.e. of the order of $30 \%$.

The coefficient $\alpha_{s}$ of the VAF long-time tail of tagged particles in a solvent, is expected to write in the low concentration limit $^{19}$

$\alpha_{s}=\frac{2}{3\left(\rho_{t}+\rho_{s} m_{s} / m_{t}\right)\left(4 \pi\left(D_{t}^{0}+\nu\right)\right)^{3 / 2}}$

where $\rho_{t}$ is the density of the solvated particles such as $\rho_{t} \rho_{s} \simeq 0$ and $D_{t}^{0}$ their diffusion coefficient, estimated in excluding the long-time tail contribution $\delta D_{t}$ to the time integral of $z(t)$ defining the full diffusion coefficient : $D_{t}=k_{B} T \int_{0}^{\infty} d t z(t) / m_{t}{ }^{13}$. This contribution is of the order of $\delta D_{t}=k_{B} T \alpha_{s} /\left(2 m_{t} t_{c}^{1 / 2}\right)$.

Since in our simulations $\rho_{t}<10^{-4}, \alpha_{s}$ is given by

$\alpha_{s}=\frac{2 m_{t}}{3 \rho_{s} m_{s}\left(4 \pi\left(D_{t}^{0}+\nu\right)\right)^{3 / 2}}$.

From this relation, it is seen that $\alpha_{s}$ depends on the solvated particle diameter only through $D_{t}^{0}$. The $D_{t}^{0}$ values are, in reduced units, $0.22,0.112,0.060$ and 0.032 for $\sigma_{00}=4,7,11$ and 17 , respectively. Since $\nu=1.71$, the $\alpha_{s}$ values are weakly depending on $D_{t}^{0}$ and, so, on $\sigma_{00}$. They are equal to $1.68,1.83,1.91$ and 1.95 ; these values must be compared to the estimates $\alpha_{s}^{f}$ of $\alpha_{s}$, obtained from the fits of $z(t)$ (cf. above and Fig. 3) on the time domain 12-18 $\tau_{0}$, equal to $8.0 \pm 0.2,3.0 \pm 0.3,1.7 \pm 0.3$ and $0.9 \pm 0.4$.

The large discrepancy between the $\alpha_{s}$ and $\alpha_{s}^{f}$ values does not seem to result from a biased estimate of $D_{t}$ due to finite size effects inherent to simulation data. The finite size effects affecting the $D_{t}$ values have been well studied in the literature ${ }^{3,20,21}$. From these works, it was concluded that a realistic estimate of the difference between the diffusion coefficient $D_{t}^{N}$ for a system of $N$ solvent particles and its value $D_{t}^{\infty}$ in the infinite system limit is given by $D_{t}^{\infty}=D_{t}^{N}+\frac{k_{B} T \xi_{c}}{6 \pi m_{s} \rho_{s} \nu L}$ 
where $\xi_{c}$ is a constant equal to $\sim 2.84$. This finite size correction is positive, independent of the solvated particle diameter and for the considered systems in our simulations has the typical value 0.005 in reduced units. So, the finite size corrections to the $D_{t}$ values computed by simulation cannot explain the difference between the two sets of $\alpha_{s}$ estimates.

In order to overcome the discrepancy between the estimates $\alpha_{s}$ and $\alpha_{s}^{f}$, we suppose that $\alpha_{s}^{f}$ scales inversely proportionaly to the nanoparticle diameter and then, that the appropriate time scale $\tau_{n}$ associated to the VAF long-time behavior of nanoparticles is not $\tau_{0}$ but is depending on the the solvated particle diameter and has the form $\tau_{n}=\sigma_{00} \tau_{0} /\left(c_{\alpha} \sigma_{11}\right), c_{\alpha}$ being constant for a given thermodynamic state of the solvent. With this hypothesis, the long-time tail of the nanoparticle VAF writes $\alpha_{s}^{f} / t^{3 / 2}=\alpha_{s} /\left(\tau_{n} t / \tau_{0}\right)^{3 / 2}$. This relation is satisfied in taking into account the estimated errors on the values of $\alpha_{s}^{f}$ and $\alpha_{s}$ for $c_{\alpha}=$ $10.5 \pm 1.0$. Fig. 4 shows that, at large times in agreement with the previous hypothesis, the VAF of nanoparticles of diameter $\sigma_{00}=4,7$ and 17 collapse within the statistical errors on the VAF of the nanoparticle with the diameter $\sigma_{00}^{r}=11$, when time is scaled proportionally to $\sigma_{00} / \sigma_{00}^{r}$.

\section{CONCLUSIONS}

The computation by MD simulations of the nanoparticle VAF demonstrates that this latter exhibits at short times a decrease which varies qualitatively when the size ratio between the nanoparticle and solvent particles increases. In addition, at large times the expected algebraic decrease proportional to $t^{-3 / 2}$ seems to be characterized by a time scale depending on the particle diameter. This last conjecture is reasonably verified by our MD data but, obviously depends on the reliability of our MD estimates of the asymptotic behavior of the nanoparticle VAF which presents clearly noticeable statistical errors as it is seen in Fig. 4. 


\section{Figure captions}

Figure 1. Normalized VAF of a solvated particle with $m_{t} / m_{s}=60$ and diameter : $\sigma_{00}=1$, (solid line and filled circle), 4 (solid line and filled square), 7 (solid line and filled diamond), 11 (solid line and filled triangle up) and 17 (solid line and filled triangle down). The statistical error is of the order of the symbol size.

Figure 2. Fit of $z(t)$ for $\sigma_{00}=1$ by an exponential. MD data : filled circle with error bars, fit : dashed line.

Figure 3. From left to right and top to bottom, fits of $z(t)$ for $\sigma_{00}=4,7,11$, and 17 for the $t / \tau_{0}$ values between 12 and 18. MD data : filled circle with error bars. Fits of the MD data with $\alpha / t^{-3 / 2}$ : solid line and with $\alpha / t^{-a_{0}}$ : dashed line.

Figure 4. Collapse of functions $z(t)$ in the time domain $10 \tau_{0}<t<18 \tau_{0}$ onto $z(t)$ for $\sigma_{00}=11$ : solid and filled square $\sigma_{00}=4$, solid line and filled diamond $\sigma_{00}=7$, solid line and triangle up $\sigma_{00}=11$ solid line triangle down $\sigma_{00}=17$. Top : unscaled functions $z(t)$, bottom : scaled functions $z(t)$. The typical statistical error on the $z(t)$ values in this time domain is \pm 0.003 . 
* Author to whom correspondance should be addressed. Electronic address: dominique.levesque@th.u-psud.fr.

1 I.W. Hamley, Introduction to Soft Matter (Wiley, New York, 2002).

2 R.I. Cukier and J.M. Deutch, Phys. Rev. 177, 240 (1989).

3 R.O. Sokolovskii, M. Thachuk and G.N. Patey, J. Chem. Phys. 125, 204502 (2006).

4 S. K. Kumar, G. Szamel and J.F. Douglas, J. Chem. Phys. 124, 214501 (2006).

5 M.G. McPhie, P.J. Daivis and I.K. Snook, Phys. Rev. E 74, 03121 (2006).

6 Song Hi Lee and R. Kapral, J. Chem. Phys. 121, 11163 (2004).

7 J.R. Schmidt and J.L. Skinner, J. Chem. Phys. 119, 8062 (2003).

8 A. McDonough, S.P. Russo and I.K. Snook, Phys. Rev. E 63, 026109 (2001).

9 F. Ould-Kaddour and D. Levesque, Phys. Rev. E 63, 011205 (2001).

10 F. Ould-Kaddour and D. Levesque, J. Chem. Phys. 118, 7888 (2003).

11 P. Español and I. Zuñiga, J. Chem. Phys. 98, 574 (1993).

12 P. Resibois and M. DeLenner, Classical Theory of Fluids (Wiley, New York, 1977).

13 J.J. Erpenbeck and W.W. Wood, Phys. Rev. A 26, 1648 (1982).

14 R.F.A. Dib, F. Ould-Kaddour and D. Levesque, Phys. Rev. E 74, 011202 (2006).

15 B. J. Alder and T. E. Wainwright, Phys. Rev. A 1, 18 (1970).

16 M. H. Ernst, E. H. Hauge, and J. M. J. van Leeuwen, Phys. Rev. Lett. 25, 1254 (1970).

17 G. L. Paul and P. N. Pusey, J. Phys. A 14, 3301 (1981).

18 V. Ya. Rudyak, G.V. Kharlamov and A.A. Belkin, High Temperature 39, 264 (2001).

19 Y. Pomeau, J. Chem. Phys. 57, 280 (1972).

20 In-Chui and Gerhard Hummer, J. Phys. Chem. B 108, 15875 (2004).

21 M. Fushiki, Phys. Rev. E 68, 021203 (2003). 


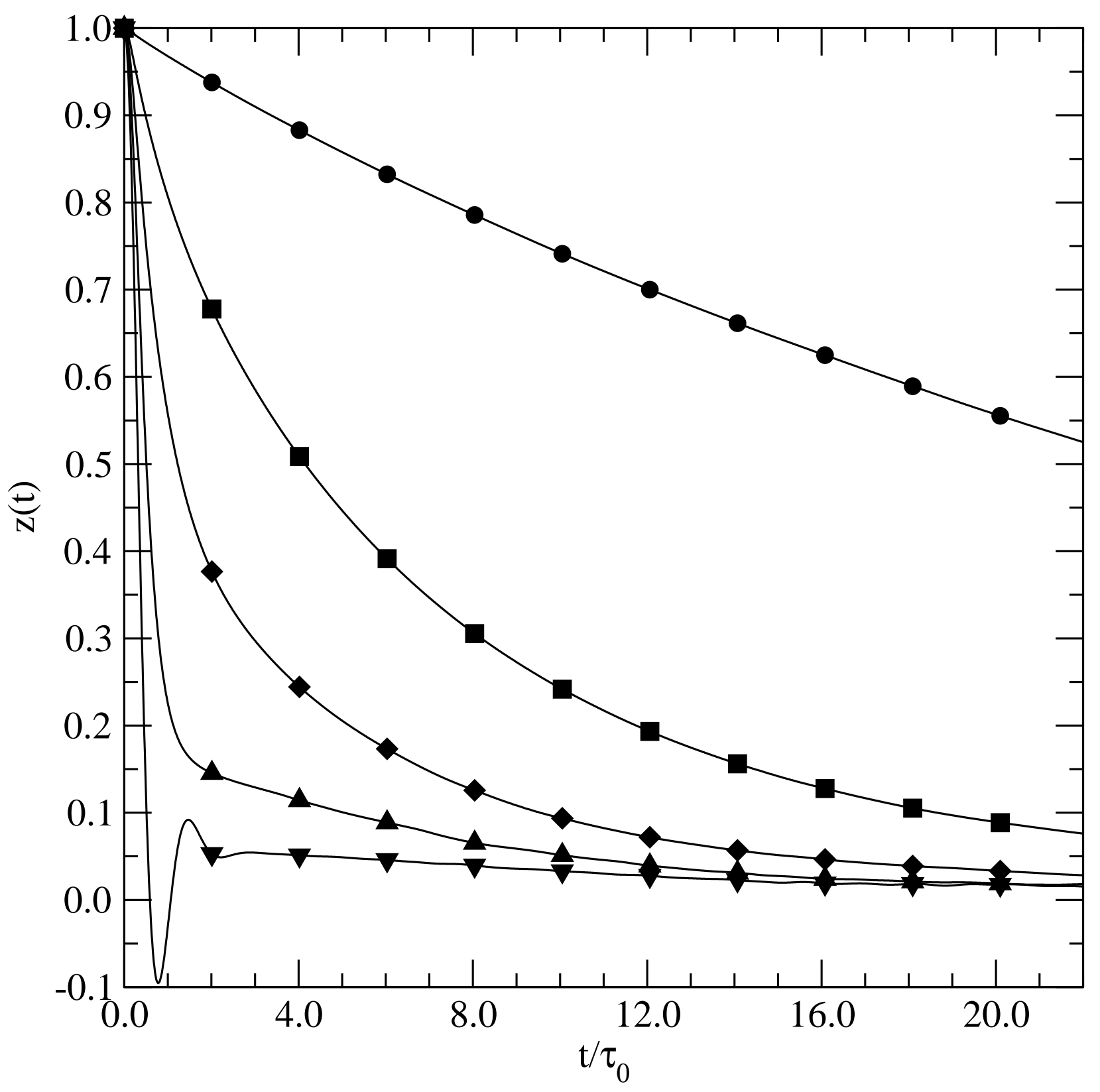

FIG. 1: 


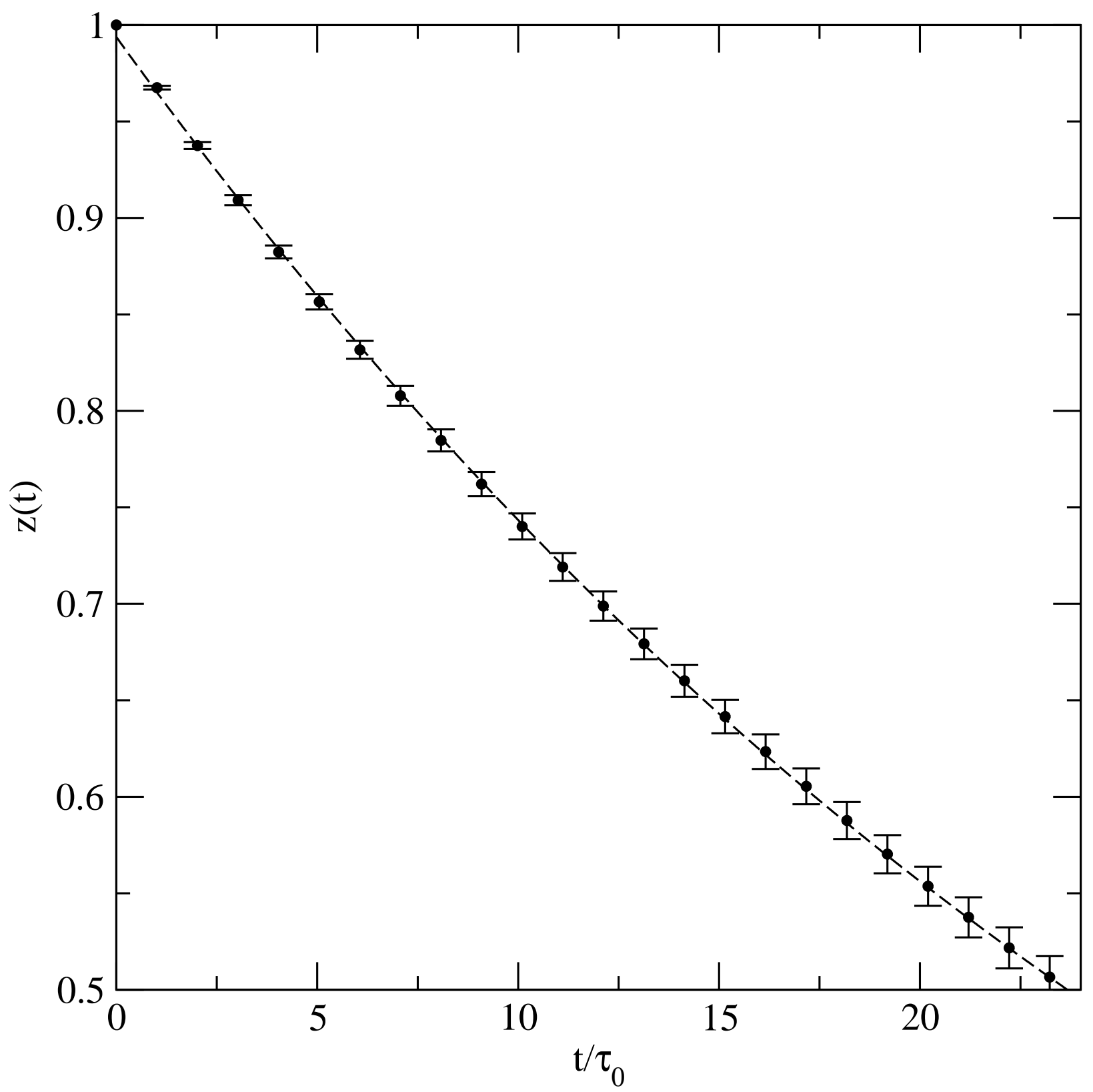

FIG. 2: 

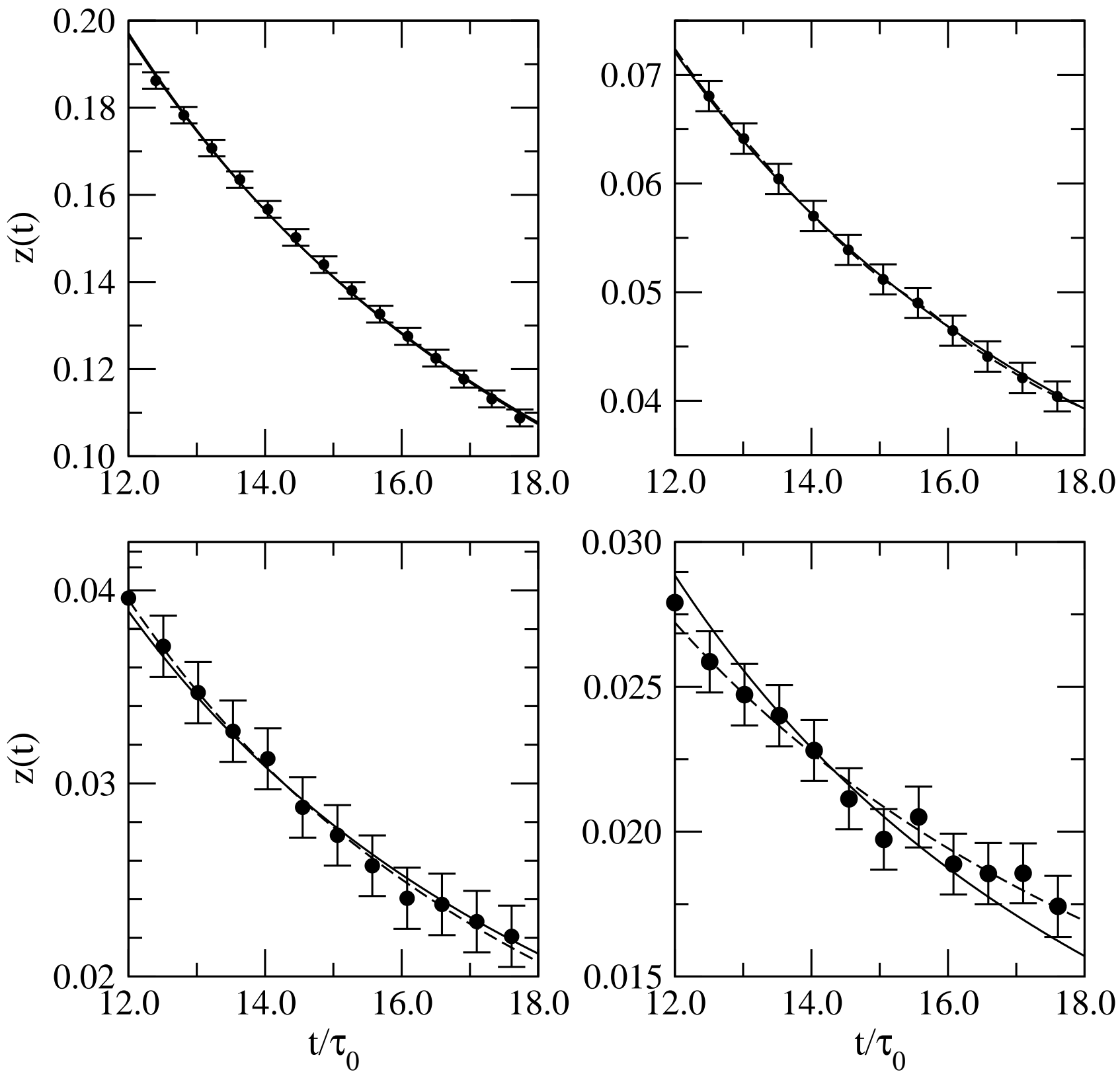

FIG. 3: 

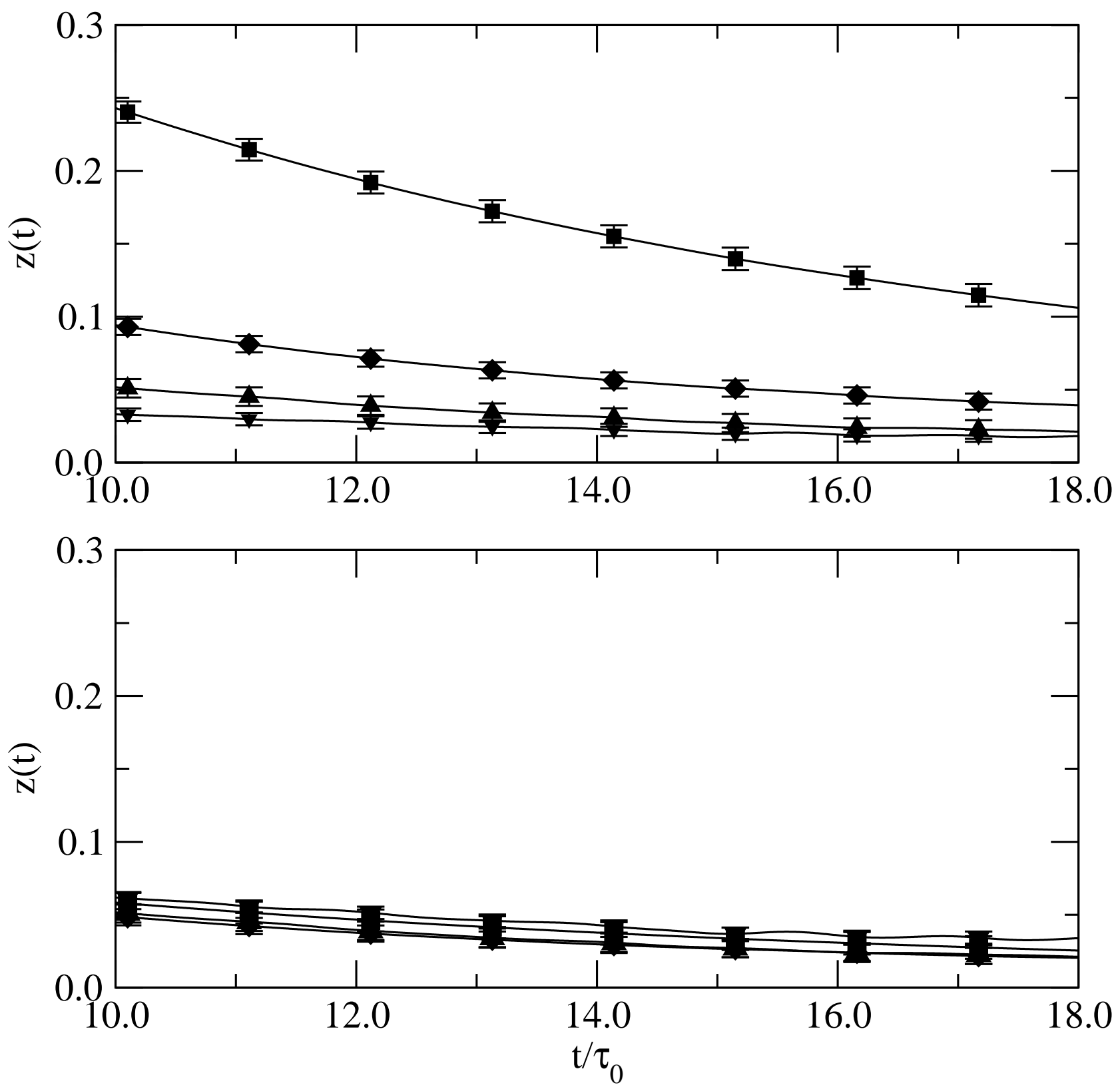

FIG. 4: 